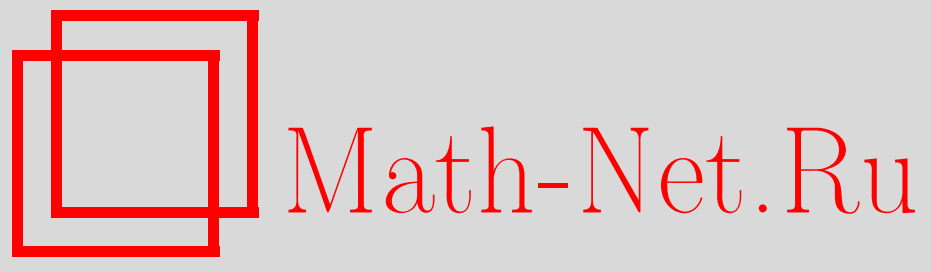

Н. Ю. Крыжановская, Ядерные оценки долгосрочной матрицы ковариаций случайного поля, $У М H, ~ 2009$, том 64, выпуск 1, 151-152

DOI: https://doi.org/10.4213/rm9263

Использование Общероссийского математического портала Math-Net.Ru подразумевает, что вы прочитали и согласны с пользовательским соглашением http://www . mathnet.ru/rus/agreement

Параметры загрузки:

IP : 54.174 .149 .18

26 апреля 2023 г., 18:26:42

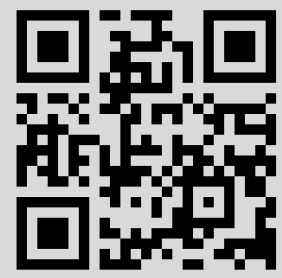




\section{Ядерные оценки долгосрочной матрицы ковариаций случайного поля}

\section{Н. Ю. Крыжановская}

Многие статистические методы основаны на состоятельных оценках долгосрочной матрицы ковариаций. Они играют важную роль в моделях регрессии, обладающих свойствами автокорреляции и гетероскедастичности (см., например, [1]). Кроме того, такие оценки исследуются в работах, связанных с обобщенным методом моментов [2], тестами на коинтеграцию и единичный корень [3]. Целый ряд статей посвящен изучению ядерных оценок долгосрочных матриц ковариаций для последовательностей зависимых случайных векторов $\left\{X_{t}\right\}_{t=1}^{\infty}$ (см. [1]-[5]). А именно, для долгосрочной матрицы ковариаций

$$
C:=\lim _{n \rightarrow \infty} \frac{1}{n} \sum_{i=1}^{n} \sum_{j=1}^{n} \mathrm{E} X_{i} X_{j}^{\top}
$$

(все элементы матрицы $C$ предполагаются конечными) рассматриваются оценки вида

$$
\widehat{C}_{n}:=\sum_{j=-n+1}^{n-1} k\left(j / \gamma_{n}\right) \widehat{\Gamma}(j),
$$

где $\widehat{\Gamma}(j):=\frac{1}{n} \sum_{t=1}^{n-j} X_{t} X_{t+j}^{\top}$ при $j \geqslant 0, \widehat{\Gamma}(j):=\frac{1}{n} \sum_{t=1-j}^{n} X_{t} X_{t+j}^{\top}$ при $j<0, k(x)$ - ядерная функция, $\gamma_{n}$ - так называемая ширина окна. Для поля $X=\left\{X_{j}, j \in \mathbb{Z}^{d}\right\}$, состоящего из случайных векторов $X_{j}=\left(X_{j, 1}, \ldots, X_{j, l}\right)^{\top}, \mathrm{E} X_{j}=0$, аналогом (1) будет матрица

$$
C:=\lim _{n \rightarrow \infty} \frac{1}{n^{d}} \sum_{i, j \in U_{n}} \mathrm{E} X_{i} X_{j}^{\top}, \quad U_{n}:=[1, n]^{d} \cap \mathbb{Z}^{d}, \quad n \in \mathbb{N} .
$$

Целью данной работы является построение оценок типа (2) в многомерном случае $(d \geqslant 1)$ и установление их слабой и сильной состоятельности.

В 80-е годы прошлого века активно исследовались оценки вида (2) с различными ядерными функциями. В [1] проводится сравнение свойств таких оценок и изучается вопрос оптимизации выбора последовательности $\left\{\gamma_{n}\right\}_{n \in \mathbb{N}}$. До появления работы Б. Хансена [4] для доказательства результатов о ядерных оценках матрицы ковариаций обычно использовали предположение о наличии конечного четвертого момента. В [4] состоятельность оценок установлена при условии конечности абсолютного момента порядка чуть большего двух. Кроме того, в [4] не требуется стационарность процесса. Если поле стационарное, то, как легко проверить, элементы матрицы $C=\left\{c_{a, b}\right\}_{a, b=1}^{l}$ имеют вид $c_{a, b}=\sum_{j \in \mathbb{Z}^{d}} \operatorname{cov}\left(X_{0, a}, X_{j, b}\right), a, b=1, \ldots, l$. Для стационарных полей существуют и другие оценки долгосрочной матрицы ковариаций [5; гл. 7].

Во многих работах результаты о ядерных оценках формулируются для $\alpha$ - и $\phi$-перемешивающих последовательностей случайных векторов. Однако проверка условий, связанных с коэффициентами перемешивания, вызывает значительные трудности (см., например, [6], [7]). В настоящей работе рассматриваются $(B L, \theta)$-зависимые случайные поля. Это понятие обобщает такие условия, как независимость, ассоциированность, положительная и отрицательная ассоциированность (см. [5]).

Итак, изучается поле (не обязательно стационарное) центрированных случайных векторов $X=\left\{X_{j}, j \in \mathbb{Z}^{d}\right\}$. Пусть все элементы матрицы $C$ (см. (3)) конечны. Будем говорить, что функция $k(\cdot)$ ядерная, если выполняются следующие условия: $k: \mathbb{R}^{d} \rightarrow[-1,1] ; k(0)=1 ; k(x)$ непрерывна для почти всех $x \in \mathbb{R}^{d}$ и непрерывна

Работа выполнена при поддержке РФФИ (грант № 07-01-00373-а). 
в нуле; $\int_{\mathbb{R}^{d}}|k(x)| d x<\infty ; k(x)=k(y)$ при $\|x\|=\|y\|$, где $\|x\|=\max _{1 \leqslant i \leqslant d}\left|x_{i}\right|$ для $x=$ $\left(x_{1}, \ldots, x_{d}\right)^{\top} \in \mathbb{R}^{d}$. Рассмотрим последовательность $\left\{\gamma_{n}\right\}_{n \in \mathbb{N}}$ такую, что $\gamma_{n} \rightarrow \infty$ при $n \rightarrow \infty$ и $\gamma_{n}^{1+2 q} n^{-1}=O(1)$ для некоторого $q>1 / 2$. Определим следующие статистики:

$$
\widehat{C}_{n}:=\sum_{j \in V_{n}} k\left(j / \gamma_{n}\right) \widehat{\Gamma}(j) ; \quad \widehat{\Gamma}(j):=\frac{1}{n^{d}} \sum_{t \in W_{j}} X_{t} X_{t+j}^{\top}, \quad j \in V_{n},
$$

где $V_{n}:=[-(n-1), n-1]^{d} \cap \mathbb{Z}^{d}, n \in \mathbb{N} ; W_{j}=W_{j}(n):=\left\{t \in U_{n}: t+j \in U_{n}\right\}, j \in V_{n}$. Эти статистики являются в некотором смысле обобщением статистик с локальным усреднением, рассматриваемых, например, в [8].

Случайное поле $X=\left\{X_{j}, j \in \mathbb{Z}^{d}\right\}$, принимающее значения в $\mathbb{R}^{l}$, называется $(B L, \theta)$-зависимъц, если существует монотонно стремящаяся к нулю при $m \rightarrow \infty$ положительная последовательность $\theta=\left\{\theta_{m}\right\}_{m \in \mathbb{N}}$ такая, что для любых конечных непересекающихся множеств $I, J \subset \mathbb{Z}^{d}$ и любых ограниченных липшицевых функций $f: \mathbb{R}^{|I| l} \rightarrow \mathbb{R}, g: \mathbb{R}^{|J| l} \rightarrow \mathbb{R}$ верно неравенство

$$
\left|\operatorname{cov}\left(f\left(X_{i}, i \in I\right), g\left(X_{j}, j \in J\right)\right)\right| \leqslant \operatorname{Lip}(f) \operatorname{Lip}(g)(|I| \wedge|J|) \theta_{\operatorname{dist}(I, J)},
$$

где $\operatorname{Lip}(f)=\sup _{u \neq v} \frac{|f(u)-f(v)|}{\|u-v\|_{*}},\|u\|_{*}=\sum_{i=1}^{|I| l}\left|u_{i}\right|, \operatorname{dist}(I, J)=\min \{\|x-y\|, x \in I, y \in J\}$ и $|I|$ - мощность множества $I$.

Пусть $\|Y\|_{p}:=\left(\sum_{a=1}^{l} \mathrm{E}\left|Y_{a}\right|^{p}\right)^{1 / p}-$ норма случайного вектора $Y=\left(Y_{1}, \ldots, Y_{l}\right)^{\top}, p>1$.

Теорема 1. Пусть $X=\left\{X_{j}, j \in \mathbb{Z}^{d}\right\}-(B L, \theta)$-зависимое векторнозначное иентрированное случайное поле, где последовательность $\theta$ такова, что $\theta_{m}=O\left(m^{-\lambda}\right) u$ $d / \lambda \in(0,1)$. Пусть $k(x)$ является ядерной функиией, а последовательность $\left\{\gamma_{n}\right\}_{n \in \mathbb{N}}$ удовлетворяет условиям, перечисленным выше, причем $q>1 / 2+d / \lambda$. Предположим, что $\sup _{j \in \mathbb{Z}^{d}}\left\|X_{j}\right\|_{p}<\infty$ для некоторого $p>\left(4 q+q^{-1}+4\right)(2 q-2 d / \lambda-1)^{-1}$. Тогда $\widehat{C}_{n} \rightarrow C$ по вероятности при $n \rightarrow \infty$.

При больших значениях $q$ можно взять $p$ достаточно близким к 2 , иначе говоря, обойтись малым запасом абсолютных моментов поля $X$.

Теорема 2. Пусть выполнены условия теоремы 1 и существует невозрастающая функция $g: \mathbb{R}_{+} \rightarrow \mathbb{R}_{+}$такая, что $|k(x)| \leqslant g(\|x\|), x \in \mathbb{R}^{d}, u \int_{\mathbb{R}^{d}} g(\|x\|) d x<\infty$.

Если к условиям теоремы 1 добавить предположение, что $k(x)$ имеет ограниченный носитель, то $\widehat{C}_{n} \rightarrow C$ п. н. при $n \rightarrow \infty$. Это следует из теоремы 2 , так как можно положить $g(z)=\mathbf{I}\{z \leqslant B\}$, где $\mathbf{I}\{G\}$ - индикатор события $G$, а $B$ - такое число, что $k(x)=0$ при $x$, для которых $\|x\|>B$.

\section{Список литературы}

[1] D.W.K. Andrews, Econometrica, 59:3 (1991), 817-858. [2] L.P. Hansen, Econometrica, 50:4 (1982), 1029-1054. [3] P. C. B. Phillips, Econometrica, 55:2 (1987), 277-301. [4] B. E. Hansen, Econometrica, 60:4 (1992), 967-972. [5] A. Bulinski, A. Shashkin, Limit theorems for associated random fields and related systems, Adv. Ser. Stat. Sci. Appl. Probab., 10, World Sci. Publ., Hackensack, NJ, 2007. [6] D. W. K. Andrews, J. Appl. Probab., 21:4 (1984), 930-934. [7] P. Ango Nze, P. Doukhan, Econometric Theory, 20:6 (2004), 995-1045. [8] A. Bulinski, N. Kryzhanovskaya, Probab. Math. Statist., 26:2 (2006), 261-281.

Н. Ю. Крыжановская (N. Yu. Kryzhanovskaya) Московский государственный университет им. М. В. Ломоносова

E-mail: natakr@gmail.com
Представлено А. В. Булинским Принято редколлегией 15.12 .2008 